\title{
A STUDY ON CARDIOVASCULAR MANIFESTATIONS OF SYSTEMIC LUPUS ERYTHEMATOSUS
}

\author{
Suresh Muthezhathu Kesavadas ${ }^{1}$, Harikrishnan Gangadharan Nair², Vijay Narayanan Harikumar ${ }^{3}$, Sandhya Somasekharan Nair ${ }^{4}$ \\ 1 Professor, Department of Internal Medicine, Government Medical College, Trivandrum. \\ 2Junior Resident, Department of Internal Medicine, Government Medical College, Trivandrum. \\ $3 J u n i o r$ Resident, Department of Internal Medicine, Government Medical College, Trivandrum. \\ ${ }^{4}$ Associate Professor, Department of Dermatology and Venereology, Government Medical College, Alleppey.
}

\section{ABSTRACT}

\section{BACKGROUND}

SLE is a classic prototype for a multisystem inflammatory disease with autoimmune aetiology. Because of the pleomorphic nature of this disease, its cardiovascular manifestations have not always been emphasised. This study aims to look at prevalence of cardiovascular manifestations in SLE.

Objective- To study the prevalence of cardiovascular manifestations in large living group of unselected patients with SLE both clinically and investigation wise including two dimensional and Doppler echocardiography and analysing their relation with clinical features of cardiovascular involvement.

\section{MATERIALS AND METHODS}

100 patients diagnosed to have SLE (Based on 2012 SLICC criteria) attending Rheumatology OPD and inpatients in General Medicine ward formed the study group. This group of patients were studied for 12 months. The study design was that of an observational study. Data was collected using pretested structured proforma. Data analysis was done using SPSS software.

\section{RESULTS}

Mean age at diagnosis of SLE was 34.5 years. Female to male ratio was 11.5:1. The most common cardiac symptom was dyspnoea on exertion (23\%). Other prominent symptoms included palpitation (11\%), chest pain (8\%), cough (7\%), syncope (3\%). Mean pulse rate was 76.7, mean systolic and diastolic blood pressures were 118.1 and 74.1 respectively. JVP was elevated in $2 \%$ of patients. Apex beat was displaced in $11 \%$ of patients. Pericardial rub was audible in 3\% of patients, S4 in $2 \%$ and S3 in $1 \%$. $8 \%$ of patients had an audible murmur, $7 \%$ had pansystolic murmur of mitral regurgitation, $1 \%$ had both MR and AR murmur. Pericardial effusion was present in $17 \%$ of patients, $13 \%$ had mild pericardial effusion whereas $4 \%$ had moderate pericardial effusion. Cardiac tamponade was not seen in any patient. Myocarditis was not seen in any patient. Left ventricular systolic dysfunction was seen in $7 \%$ whereas diastolic dysfunction was more common with a prevalence of $10 \%$. Mitral regurgitation was the most common valvular lesion identified, with a prevalence of $10 \%$. The prevalence of aortic regurgitation was 3 . Pulmonary hypertension was found with a prevalence of $6 \%$. Libman-Sacks endocarditis was not observed.

\section{CONCLUSION}

Prevalence of cardiovascular manifestation in SLE in our study was 37\%, the most common manifestations being Pericarditis and Pericardial effusion-17\%. The most common valvular lesion identified was Mitral Regurgitation (10\%). A high index of clinical suspicion is needed to pick up cardiac lesions in SLE. Though many of these patients are asymptomatic, early intervention can be beneficial.

\section{KEYWORDS}

SLE, Pericarditis, Pericardial Effusion, Mitral Regurgitation.

HOW TO CITE THIS ARTICLE: Kesavadas SM, Nair HG, Harikumar VN, et al. A study on cardiovascular manifestations of systemic lupus erythematosus. J. Evolution Med. Dent. Sci. 2017;6(41):3223-3226, DOI: 10.14260/Jemds/2017/698

\section{BACKGROUND}

Systemic Lupus Erythematosus is one of the most common autoimmune systemic disease with a prevalence of $500 /$ million population and an annual incidence of 50 to $70 /$ million.(1) SLE can virtually involve any system in the human body.

One of the leading causes of morbidity and mortality in SLE is due to cardiovascular involvement. SLE can involve all

Financial or Other, Competing Interest: None.

Submission 15-04-2017, Peer Review 11-05-2017,

Acceptance 17-05-2017, Published 22-05-2017.

Corresponding Author:

Dr. Vijay Narayanan Harikumar,

Prashanth, NRA-27, Navarangam Lane,

Medical College P.O. Trivandrum-11, Kerala.

E-mail: vijaynh@gmail.com

DOI: $10.14260 /$ jemds $/ 2017 / 698$ the three layers of the heart (Pericardium, myocardium and endocardium).

Cardiovascular disease in SLE was first described by Kaposi in 1872. SLE has a wide spectrum of cardiovascular manifestations. Premature atherosclerosis, systolic and diastolic dysfunction, pulmonary hypertension and involvement of conduction system are well described entities in SLE. The prevalence of cardiovascular manifestations varies among different studies with an average estimate of about $30-50 \%$. Causation is often multifactorial with both SLE related inflammatory mediators and atherosclerosis being held responsible for cardiac involvement.

Cardiovascular disease is clinically silent in vast majority of the patients but occasionally patients can present with florid symptoms like rapidly progressive heart failure. The subclinical nature of cardiovascular disease is best understood by the fact that the prevalence of cardiac lesions is higher in autopsy series. 
In clinical practice, it is difficult to differentiate cardiovascular manifestations which are primarily due to SLE from other causes like systemic hypertension, anaemia, infections and renal failure. In addition, the majority of cardiac lesions are clinically silent. Therefore, a high index of suspicion is required for early diagnosis of various cardiac lesions in SLE.

\section{MATERIALS AND METHODS}

The study was conducted among patients attending Rheumatology Clinic and those admitted in Internal Medicine wards of Government Medical College, Trivandrum. SLE was diagnosed based on 2012 SLICC criteria.

\section{Sample Size}

Desired sample size was calculated using the formula.

$\mathrm{N}=\frac{\mathrm{z}^{2} 1-\alpha / 2 p(1-p)}{\delta^{2}}$

According to a reference study "A study of cardiovascular manifestations in SLE in upper Assam", the overall prevalence of cardiovascular manifestation was $49.2 \%$.

$\mathrm{P}=49.2 \%$.

$\delta=$ Relative Precision ( $20 \%$ of P).

$1-(\alpha / 2)=$ Desired confidence level $(95 \%)$.

Sample size (N) was calculated to be 99 .

\section{Inclusion and Exclusion Criteria}

100 patients satisfying the 2012 SLICC criteria for SLE and those who gave written informed consent were included in the study.

Patients with diabetes mellitus, systemic hypertension, dyslipidaemia and other risk factors for cardiovascular diseases were excluded.

\section{Method of Data Collection}

Those who were diagnosed as SLE were evaluated clinically by detailed history taking and clinical examination with the help of a structured questionnaire. Relevant investigations including electrocardiogram, two-dimensional echocardiogram and Doppler echocardiography were done.

\section{RESULTS}

Of the 100 SLE patients, females constitute $92 \%$ and males were $8 \%$, the youngest patient was 13 years of age while the oldest patient included in the study was 78 years old. Mean age of SLE patients in the study was 34.5 years. $30 \%$ of the patients were in the age group of $21-30$ and $21 \%$ were in the age group of 31-40. The male: female ratio was 1:11.5.

\begin{tabular}{|c|c|c|c|c|c|}
\hline & N & Minimum & Maximum & Mean & SD \\
\hline Age (Years) & 100 & 13 & 78 & 34.5 & 13.6 \\
\hline Pulse & 100 & 54 & 100 & 76.7 & 10.6 \\
\hline Systolic BP & 100 & 90 & 140 & 118.1 & 13.8 \\
\hline Diastolic BP & 100 & 60 & 90 & 74.7 & 7.0 \\
\hline \multicolumn{5}{|c|}{ Table 1. Distribution of Age, Pulse } \\
Rate, Systolic and Diastolic Blood Pressure \\
\hline
\end{tabular}

\section{Symptoms}

Chest Pain suggestive of Pericardial Pain was seen in $8 \%$ of patients. $23 \%$ patients had dyspnoea on exertion. Cough as a symptom was present in $7 \%$ of patients. Palpitation was seen in $11 \%$ of SLE patients. Palpitation was more commonly seen in patients with valvular lesions (Mitral or Aortic Regurgitation). Syncope was seen in $3 \%$ of patients.

\section{Signs}

JVP was elevated in $2 \%$ of patients. Apex beat was displaced in $11 \%$ of patients. When displaced, the apical impulse was suggestive of left ventricular hypertrophy.

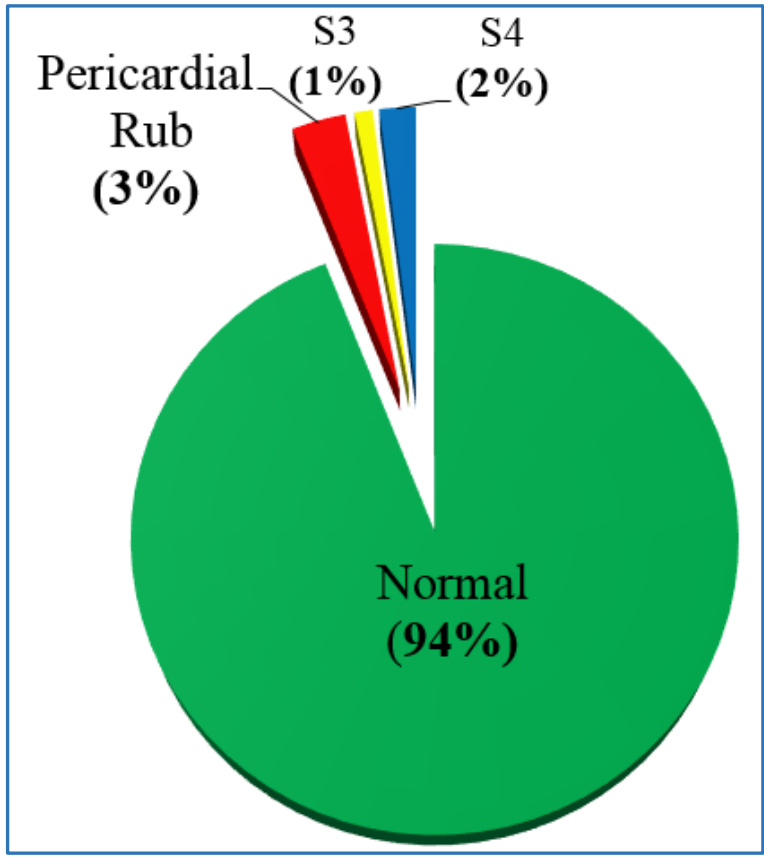

Figure 1. Heart Sounds in SLE

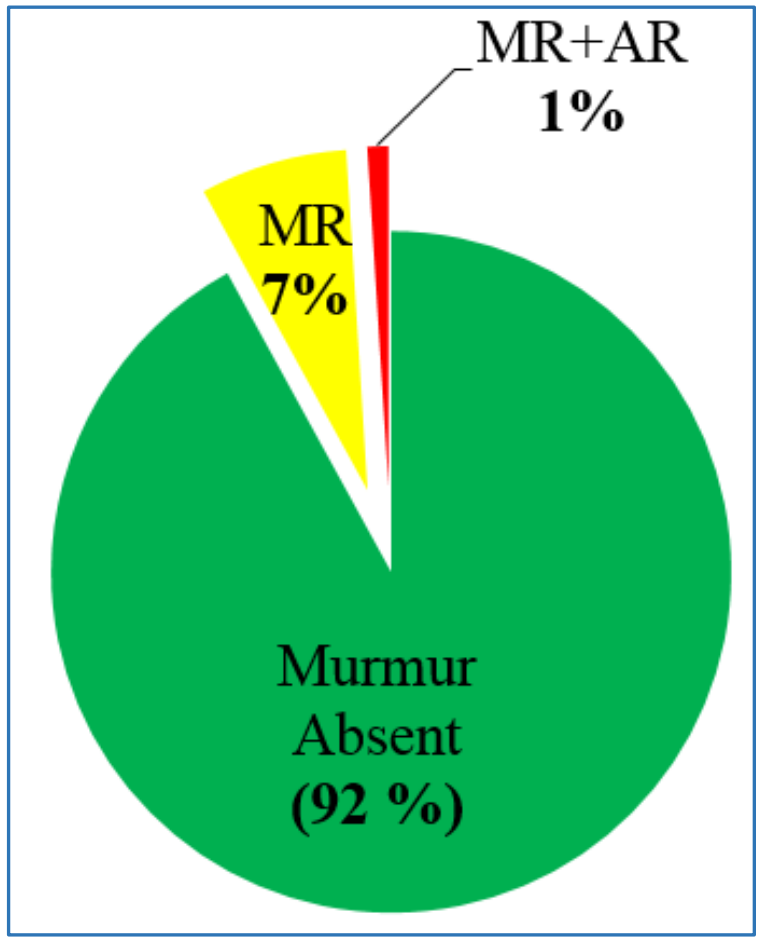

Figure 2. Murmur in SLE

\section{Cardiac Lesions in SLE}

Pericardial effusion was the single most common cardiac lesion seen in $17 \%$ of patients. 


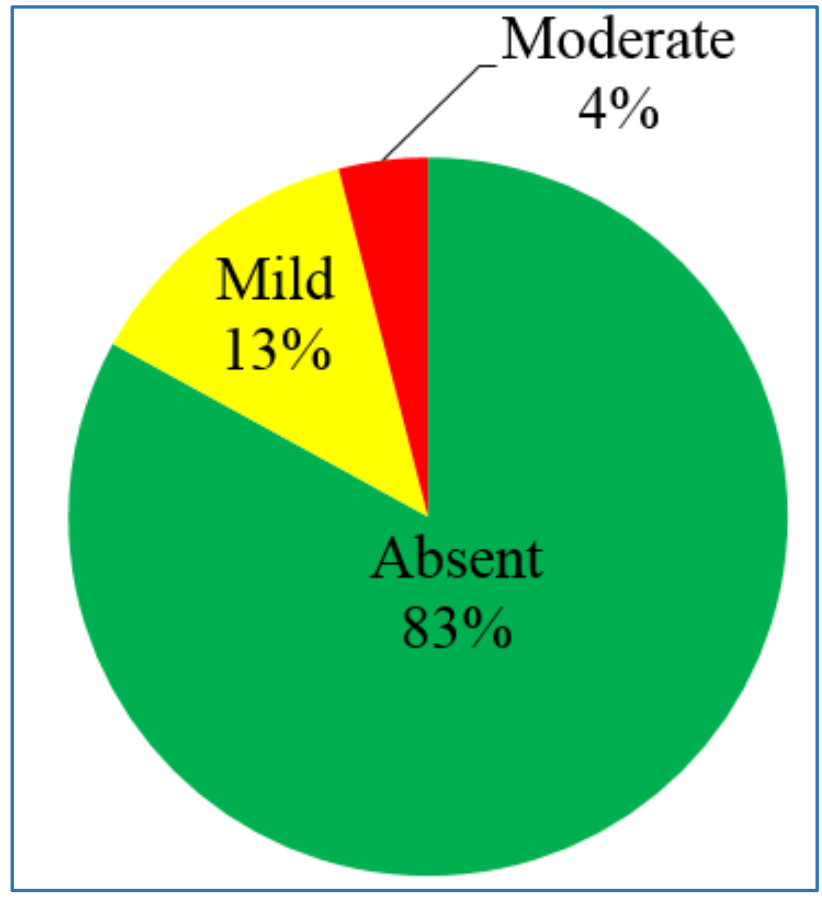

Figure 3. Pericardial Effusion

Left ventricular systolic dysfunction was seen in $7 \%$ while the prevalence of diastolic dysfunction was $10 \%$.

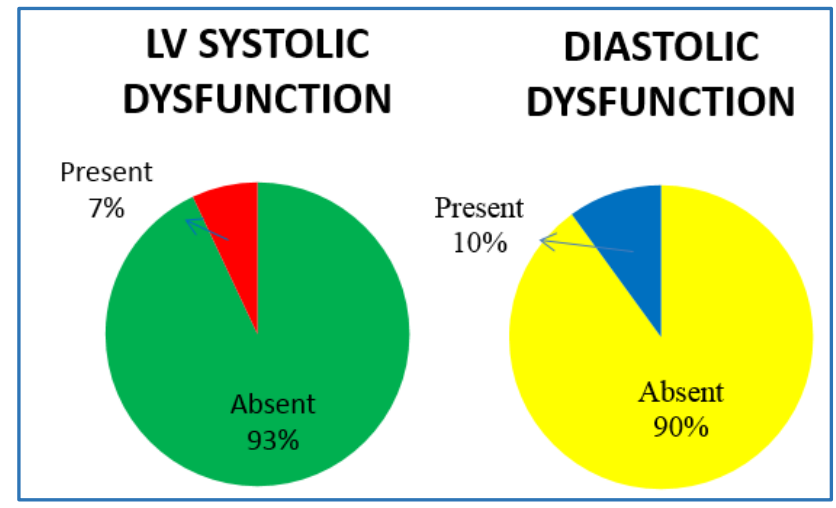

Among valvular lesions, Mitral Regurgitation was seen in $10 \%$ SLE patients while Aortic Regurgitation was seen in $3 \%$ of patients.

Pulmonary Hypertension was seen in $6 \%$ of the study population.

Libman-Sacks Endocarditis was not seen in any patient in the study group.

\section{DISCUSSION}

Cardiovascular involvement is one of the leading causes for morbidity and mortality in SLE. SLE can involve all the three layers of the heart as well as pulmonary and systemic vasculature and the conducting system of the heart. This study aimed to find the prevalence of cardiovascular manifestations in SLE.

In this study, 100 SLE patients were studied over 1 year period. The mean age of the study population was 34.5 years and $30 \%$ of the patients were in the age group of $21-30$ and $21 \%$ in the age group of 31-40. The male: female ratio was 1:11.5. According to a study by Malaviya et al, Mean age of onset in Indian SLE is 24.5 years and male: female ratio is $1: 11 .(2)$

A relevant clinical history was elicited in all the SLE patients included in our study. The most common symptom was dyspnoea on exertion, present in $23 \%$ of patients. Palpitation was the second most common symptom (11\%) followed by pleuropericardial pain (8\%), cough (7\%) and syncope (3\%). In a study conducted at RIMS Imphal by Subba et al,(3) shortness of breath was found in $41 \%$ of patients followed by palpitation in $19.7 \%$ and orthopnoea in $16.2 \%$.

Physical examination revealed pulse and blood pressure values were in a range not different from the general population (mean Pulse Rate -76.7, mean Systolic BP -118.1, mean Diastolic BP- 74.7). JVP was elevated in only $2 \%$ of the patients and the apical impulse was displaced in 11\%. Heart sounds were normal in a majority of patients (94\%) whereas $3 \%$ had an audible pericardial knock, S4 was heard in 2\% and $1 \%$ had S3. Pansystolic murmur of Mitral Regurgitation was made out by auscultation in $7 \%$ of patients while $1 \%$ had murmurs of both mitral and aortic regurgitation.

Pericardial Effusion was the single most common cardiovascular manifestation with a prevalence of $17 \%$. Moderate pericardial effusion was seen in $4 \%$ while $13 \%$ had mild pericardial effusion. While majority of patients with effusion are asymptomatic, a few complained of dyspnoea on exertion and palpitation. Petri et al ${ }^{(4)}$ described a prevalence of $12-47 \%$ of pericardial effusion in SLE patients. In a study conducted by Kotokey et al(5) amongst SLE patients in upper Assam, pericardial effusion was the most common cardiac lesion with a prevalence of $21.92 \%$.

Amongst valvular heart disease, mitral regurgitation was the most common lesion seen in $10 \%$ of patients. Mitral Regurgitation was mild in severity in almost all the cases and most patients were asymptomatic. When symptomatic, palpitation was the most common symptom found in $20 \%$ of patients with Mitral regurgitation. Cervera et al(6) did a prospective study on 70 patients with SLE and found that mitral valve abnormalities were found in 33\% of patients. Similar to our study, Mitral regurgitation was the most common valvular lesion identified and majority of cases were mild in severity.

In our study, Aortic Regurgitation was seen in 3\% of patients while it was $9.1 \%$ in the study by Cervera et al.(6) In both the studies, the majority of cases were mild in severity and clinically asymptomatic.

Prevalence of Left Ventricular dysfunction in our study was $7 \%$ and that of diastolic dysfunction was $10 \%$. Systolic dysfunction was mild, and most common symptom in this group was dyspnoea on exertion. Patients with diastolic dysfunction had stage 1 diastolic dysfunction and all of them were clinically asymptomatic. Kotokey et al(5) found a prevalence of $1.37 \%$ for systolic dysfunction and $2.4 \%$ for diastolic dysfunction. In the study by Subba et al(3) the prevalence of mild systolic dysfunction was $29.5 \%$, moderate systolic dysfunction was $7.7 \%$ and diastolic dysfunction was $11.5 \%$. The disparity in results as compared to our study could be due to the fact that patients with systemic hypertension, diabetes, dyslipidaemia and other risk factors for cardiovascular disease were excluded from our study.

Prevalence of Pulmonary hypertension was $6 \%$ in our study population. Pulmonary hypertension was a mild and incidental finding during transthoracic echocardiogram and 
was more commonly seen with a long duration of disease (mean duration of illness of 5 years or more). The prevalence of pulmonary hypertension was $5.48 \%$ in the study by Kotokey et $\mathrm{al}^{(5)}$ whereas it was seen in $8.2 \%$ according to Akdogan et al.(7)

No cases of myocarditis or Libman-Sacks endocarditis was encountered in our study. Though considered characteristic of SLE, the incidence of Libman-Sacks endocarditis has come down significantly in the postcorticosteroid era. Similar to our study, Kotokey et al(5) did not come across any case of Libman-Sacks endocarditis in their study. An autopsy study was conducted by Panchal et $\mathrm{al}^{(8)}$ to find out cardiovascular involvement in SLE and only one case of Libman-Sacks Endocarditis was found.

\section{CONCLUSION}

Cardiovascular disease in SLE is an underdiagnosed entity as most of the lesions are clinically silent. The total prevalence of cardiovascular manifestations was 37\%. Pericardial Effusion was the most common cardiac lesion with a prevalence of $17 \%$. Mitral valve is the most commonly affected valve followed by aortic valve. Regurgitant lesions were found to be more common than stenotic lesions.

Prevalence of Mitral Regurgitation was 10\% whereas that of Aortic regurgitation was 3\%.

Diastolic dysfunction is more common than systolic dysfunction $(10 \%$ vs. $7 \%$ respectively). Pulmonary Hypertension was seen with a prevalence of $6 \%$. No cases of Libman-Sacks Endocarditis or Lupus Myocarditis were encountered in our study.

The addition of Transthoracic Echocardiogram in the routine evaluation of SLE patients can help in increasing the detection of cardiovascular lesions.

\section{REFERENCES}

[1] Klippel JH. Systemic lupus erythematosus: demographics, prognosis, and outcome. J Rheumatol Suppl 1997;48:67-71.

[2] Malaviya AN, Singh RR, Singh YN, et al. Prevalence of systemic lupus erythematosus in India. Lupus 1993;2(2):115-8.

[3] Subba S. Cardiovascular manifestations in patients with systemic lupus erythematosus. IOSR J of Dent and Med Sci IOSR-JDMS 2015;14(7):50-4.

[4] Petri M. Cardiovascular systemic lupus erythematosus. 2004:913-42. Available from: https://jhu.pure.elsevier.com/en/publications/cardiova scular-systemic-lupus-erythematosus-3

[5] Kotokey RK, Rajkhowa K, Chaliha MS, et al. A study of cardiovascular manifestations in systematic lupus erythematosus in upper Assam. J Indian Coll Cardiol 2012;2(1):29-32.

[6] Cervera R, Font J, Pare C, et al. Cardiac disease in systemic lupus erythematosus: prospective study of 70 patients. Ann Rheum Dis 1992;51(2):156-9.

[7] Akdogan A, Kilic L, Dogan I, et al. Pulmonary hypertension in systemic lupus erythematosus: pulmonary thromboembolism is the leading cause. J Clin Rheumatol 2013;19(8):421-5.

[8] Panchal L, Divate S, Vaideeswar P, et al. Cardiovascular involvement in systemic lupus erythematosus: an autopsy study of 27 patients in India. J Postgrad Med 2006;52(1):5-10. 